\title{
Anforderungen von TARMED an die EDV
}

\author{
W. Häuptli
}

TARMED wird kaum wesentliche neue Anforderungen an die EDV stellen. Nebst all dem, was EDV schon heute meistert, sollte künftig die Möglichkeit zum elektronischen Nachführen von Änderungen des Tarifs und zum elektronischen Datenaustausch zur Standardversion gehören. Die EDV-Ausrüstung der Arztpraxen bis zur geplanten Einführung von TARMED sollte eigentlich problemlos gelingen, vor allem wenn die längst vorhandenen Möglichkeiten zu Vorarbeiten genutzt wurden. konforme Abrechnung garantieren soll. Doch die Installation derselben ist kaum notwendig. Wer sein Tarifpositionsspektrum einmal kennt, wird im $\mathrm{Nu}$ auch mit den zugehörenden Abrechnungsmodulationen vertraut sein. Ist aber trotzdem Gewissheit gefragt, so wird sich bestimmt noch externe Überprüfung anbieten. Damit ist die Notwendigkeit der Installation eines sogenannten Validators mehr als fraglich, selbst wenn dieser unentgeltlich sein sollte. Denn er benötigt ansehnlich Speicherplatz und hohe Rechnerleistung, stellt also hohe Ansprüche auch an die Hardware. Neue Tarifversionen dürften Änderungen des Validators nach sich ziehen. Und schliesslich könnte in Anbetracht der raschen EDV-Entwicklung möglicherweise schon nach wenigen Jahren Inkompatibilität der Systeme eine umfassende Erneuerung erforderlich machen. Dies hat zurzeit nicht absehbare Kosten zur Folge. Auch deshalb dürfte es sich lohnen, zu prüfen, ob nicht einfachere oder externe Kontrollen genügen.

Die Rechnungen unter TARMED werden sich nur unwesentlich von den heutigen unterscheiden. Der genaue obligatorische Rechnungsinhalt ist in den bilateralen Verträgen zwischen Leistungserbringer und Versicherer festgehalten. Er differiert leicht zwischen KV- und UV-/MV-/ IV-Bereich. Neu ist die EAN-Nummer zwecks Identifikation des Arztes in bezug auf seine Dignität und anerkannten Sparten aufzuführen. Da bislang kein einheitliches Rechnungsformular vereinbart ist, bestehen keine Auflagen bezüglich Rechnungstopologie.

Den elektronischen Datenaustausch gilt es zu fördern, denn zwei Jahre nach Einführung von TARMED muss die Abrechnung elektronisch erfolgen. Für den UV-/MV-/IV-Bereich ist dazu der XML-Standard vertraglich vereinbart. Für den KV-Bereich fehlen entsprechende Spezifikationen.

TARMED wird also kaum wesentliche neue Anforderungen an die EDV stellen. Nebst all dem, was EDV schon heute meistert, sollte künftig die Möglichkeit zum elektronischen Nachführen von Änderungen des Tarifs und zum elektronischen Datenaustausch, sei es mit Versicherer oder Datensammelstelle, zur Standardversion gehören. Damit dürfte die EDV-Ausrüstung der Arztpraxen bis zur geplanten Einführung von TARMED gelingen, um so problemloser jenen, welche die längst vorhandenen Möglichkeiten zu Vorarbeiten genutzt haben. 